\section{Outbreak of Ralstonia pickettii Pseudobacteremia Among Patients With Hematological Malignancies}

TO THE EDITOR-Ralstonia pickettii (formerly named Burkholderia pickettii) is a nonfermentative gram-negative bacillus isolated from environmental sources and infrequently from clinical samples. We report here on a outbreak of pseudobacteremia in immunocompromised patients hospitalized in the 31-bed department of hematology in our hospital in (Hôpital Saint-Antoine, Paris, France).

In March and April 2005, six patients with hematological malignancies who were hospitalized in laminar air-flow rooms developed bacteremia due to $R$. pickettii. This bacterium had not been isolated in our 760-bed hospital in the preceding year. Clinical and microbiological features of each case patient are reported in the Table. All the patients were hospitalized in different rooms on the same unit, and all had a central venous catheter in place. After microbiological test results were obtained, only 2 patients were specifically treated with parenteral antimicrobial therapy.

Immediately after the recognition of this outbreak, microbiological investigations were undertaken. Cultures for $R$. pickettii were performed with multiple specimens, including the antiseptics used for preparation of the skin (6 samples cultured), sterile saline vials (6 samples), a heparin preparation ( 1 sample), plain and antiseptic soaps (4 samples), alcohol hand-rub preparation (3 samples), microfiltered tap water ( 33 samples), water from the sink drains (19 samples), and unopened blood culture bottles from the same batches used for culture of blood samples from the case patients (10 samples). A 1-week observational study of clinical practices inside the department of hematology was also performed by a trained infection control nurse to better understand the possible routes of contamination. After this study, 6 samples from the main 8 - $\mathrm{L}$ tank and $500-\mathrm{mL}$ bottles of detergentdisinfectant solution (didecyl dimethyl ammonium 5\%, ethylen diamine tetracetic acid 15\%, alcohol lauric acid ethoxylated $7 \%$, and aminopropyl laurylamin 17\% [Surfanios; Anios]) were cultured. Environmental swab samples were plated on trypticase soy agar (Oxoid). For liquid samples (eg, detergent-disinfectant solution, alcohol hand-rub preparation, and microfiltered water), $50-\mathrm{mL}$ samples were filtered through a $0.45-\mu \mathrm{m}$ pore size filter that was placed on trypticase soy agar (Millipore).

All the strains recovered from the environment and from patients were compared by pulsed-field gel electrophoresis using the SpeI macrorestriction enzyme (GenePath; BioRad). ${ }^{1}$ The antimicrobial susceptibility patterns of the strains were also determined using the disk diffusion method on MuellerHinton agar (Bio-Rad). ${ }^{2}$ We also tested $R$. pickettii isolates for their in vitro potential resistance to the detergent-disinfectant solution, according to the French standard T72-300. ${ }^{3}$

Pseudobacteremia was rapidly suspected for the following reasons: (1) 4 of the 6 affected patients had no clinical signs of septicemia (blood cultures were performed systematically every week according to the ward protocol); (2) there was no sign of local infection at the insertion site of the central venous catheters in these 6 patients, and culture of catheter tips yielded no pathogens in 2 cases; and (3) throat and stool specimens that were routinely obtained and cultured twice weekly yielded no pathogens, suggesting that patients were not colonized with $R$. pickettii.

Cultures of environmental specimens (eg, water, soaps, antiseptics, and unopened blood culture bottles) were negative for $R$. pickettii. The observational study of clinical practices showed that nurses routinely disinfected the external surface of a blood culture bottles with a detergent-disinfectant solution (Surfanios; Anios) just before taking it into the laminar air-flow rooms for collection of a blood sample. This procedure had never been validated by the infection control committee of the hospital. The detergent-disinfectant solution

TABLE. Clinical Features of Patients With Pseudobacteremia Due to Ralstonia pickettii

\begin{tabular}{|c|c|c|c|c|c|c|c|c|}
\hline Patient & Sex, age & $\begin{array}{c}\text { Underlying } \\
\text { disease(s) }\end{array}$ & $\begin{array}{c}\text { Time of onset of } \\
\text { pseudobacteremia, } \\
\text { days since } \\
\text { admission }\end{array}$ & $\begin{array}{l}\text { Proportion of } \\
\text { blood cultures } \\
\text { positive for } \\
\text { R. pickettii } \\
\end{array}$ & $\begin{array}{l}\text { Aplasia } \\
\text { present }\end{array}$ & $\begin{array}{c}\text { Temperature, } \\
{ }^{\circ} \mathrm{C} \\
\end{array}$ & $\begin{array}{c}\text { Blood } \\
\text { transfusion } \\
\text { received }\end{array}$ & $\begin{array}{c}\text { Chemotherapy } \\
\text { received }\end{array}$ \\
\hline 1 & $M, 48$ & AML, FAB4 & 11 & $2 / 9$ & No & $37.7^{\mathrm{a}}$ & No & Yes \\
\hline 2 & $M, 56$ & AML, FAB4 & 22 & $1 / 18$ & Yes & 36.6 & Yes & Yes \\
\hline 3 & $M, 52$ & $\mathrm{AML}, \mathrm{FAB} 4$ & 10 & $1 / 10$ & Yes & 37 & No & Yes \\
\hline 6 & $M, 39$ & AML, FAB4 & 2 & $5 / 12$ & No & 37 & No & No \\
\hline
\end{tabular}

Noт E. AML, acute myeloblastic leukemia; FAB, French-American-British classification; NHL, non-Hodgkin lymphoma.

With shivers.

b The day after $R$, pickettii was detected in blood culture. 
they used was prepared in the nurse office (by dilution of 20 $\mathrm{mL}$ of the detergent-disinfectant in $8 \mathrm{~L}$ of tap water) every morning in an $8-\mathrm{L}$ tank, and then $500-\mathrm{mL}$ bottles were filled from this tank and placed in each patient's room. Bacteriological analysis of detergent-disinfectant solution samples taken from the 8-liter tank and from 2 different $500-\mathrm{mL}$ bottles showed contamination with $R$. pickettii. This bacterium was also recovered from the external surface of the tap, from the opening receptacle of the 8-L tank of detergentdisinfectant, and from the external surfaces of 2 blood culture bottles in which $R$. pickettii had been isolated.

All the strains isolated from the patients and the environment had identical patterns on pulsed-field gel electrophoresis (Figure). They shared the same pattern of resistance to polymyxin, fosfomycin, fluoroquinolones, aminoglycosides (gentamicin, netilmicin, tobramycin, and amikacin), cefalothin, cefoxitin, and cefepim. They were susceptible to amoxicillin-clavulanate, piperacillin-tazobactam, trimethoprimsulfamethoxazole, and imipenem. Results of in vitro studies showed that the $R$. pickettii strains isolated were fully susceptible to the detergent-disinfectant.

As soon as this route of transmission was identified, the detergent-disinfectant solution tanks and bottles were immediately withdrawn from the ward. The procedure for handling and inoculating blood culture bottles was revised, and use of a alcohol-based disinfectant agent was strongly recommended by the infection control committee. In the following 2 months, no further cases occurred.
Our investigations revealed contamination of detergentdisinfectant solution used to clean the external surface of blood culture bottles before their introduction into the laminar air-flow room. We hypothesized that the wiping of the external surface of blood culture bottles with this contaminated detergent-disinfectant solution probably led to the contamination of the receptacle of the blood culture bottles and the subsequent introduction of this bacterium into the bottle. This hypothesis was confirmed by the recovery of $R$. pickettii from external surfaces of 2 blood culture bottles in which $R$. pickettii had been isolated. Nevertheless, the origin of the contamination of the detergent-disinfectant solution remains unclear. We cannot explain why the isolated strains were susceptible in vitro to the disinfectant but were isolated from the disinfectant solution in use. Actually, 2 possibilities were considered: the presence of visible limestone deposits and biofilm inside the 8-L tank and around the tap could have promoted survival of the bacteria, or an inappropriate dilution of the detergent-disinfectant could have led to a concentration of active substance less than that recommended by the manufacturer.

This is the first report of an outbreak of pseudobacteremia due to $R$. pickettii traced to the use of contaminated detergentdisinfectant. Outbreaks of pseudobacteremia have been previously described by Verschragen et $\mathrm{al}^{4}$ (an outbreak in 17 patients from different wards) and Maroye et al. ${ }^{5}$ (an outbreak in 6 pediatric patients). In both cases, the common source was the aqueous chlorhexidine solution prepared by the hospital

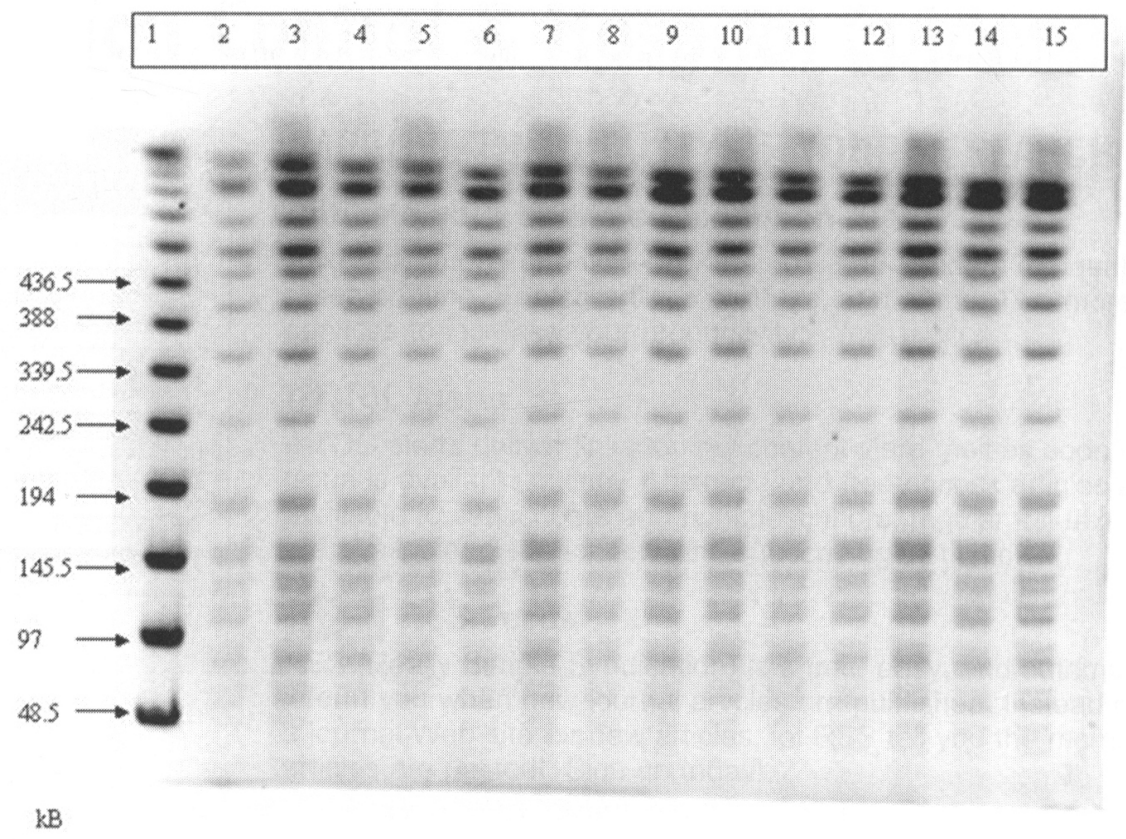

FIGURE. Pulsed-field gel electrophoresis patterns for Ralstonia pickettii strains isolated from patients (lanes 2-7) and from the environment (lanes 8-15). Lane 1, $\lambda$ ladder; lanes 2-7, strains from patients; lanes 8-13, environment (eg, detergent-disinfectant solution and the main tank and $500-\mathrm{mL}$ bottles that held the solution); lanes 14 and 15 , samples from external surfaces of blood culture bottles in which $R$. pickettii had been isolated. 
pharmacy with contaminated distilled water. In another study, an outbreak of pseudobacteremia caused by $R$. pickettii was associated with a breakdown of aseptic blood culture technique that involved the use of contaminated citrated bottles. ${ }^{6}$

If a pathogen is repeatedly isolated, infection control practitioners must promptly start investigations to determine the origin of the microorganism. The present investigation of an outbreak of pseudobacteremia reemphasizes the importance of strict adherence to the aseptic blood culture technique and the need for regular quality control evaluation of this procedure.

\section{ACKNOWLEDGMENT}

We thank all the medical and nonmedical staff of the hematology department for their help and availability.

F. Barbut, PharmD, PhD; M.-J. Kosmann, RN; V. Lalande, PharmD; D. Neyme, BSc; P. Coppo, MD, PhD; N. C. Gorin, MD, PhD

From the Infection Control Unit (F.B., M.J.K., D.N.), the Department of Microbiology (V.L.), and the Department of Hematology (P.C., N.C.G.), Saint-Antoine Hospital, Assistance Publique-Hôpitaux de Paris, Paris, France.
Address reprint requests to Frédéric Barbut, MD, UHLIN, Hôpital SaintAntoine, 184 Rue du Faubourg Saint-Antoine, 75012 Paris (frederic.barbut@ sat.ap-hop-paris.fr).

Infect Control Hosp Epidemiol 2006; 27:642-644

(C) 2006 by The Society for Healthcare Epidemiology of America. All rights reserved. 0899-823X/2006/2706-0021\$15.00.

\section{REFERENCES}

1. Chetoui H, Melin P, Struelens MJ, et al. Comparison of biotyping, ribotyping, and pulsed-field gel electrophoresis for investigation of a common-source outbreak of Burkholderia pickettii bacteremia. J Clin Microbiol 1997; 35:1398-1403.

2. Soussy CJ. Comité de l'Antibiogramme de la Société Française de Microbiologie report 2003. Int J Antimicrob Agents 2003; 21:364-391.

3. Association Frnacaise de Normalisation (AFNOR). T72-300: antiseptiques et désinfectants utilisés à l'état liquide, miscibles à l'eau: essai de suspension par dilution-neutralisation-détermination de l'efficacité des produits sur divers microorganismes dans les conditions pratiques d'emploi. AFNOR; 1989.

4. Maroye P, Doermann HP, Rogues AM, Gachie JP, Megraud F. Investigation of an outbreak of Ralstonia pickettii in a paediatric hospital by RAPD. I Hosp Infect 2000; 44:267-272.

5. Verschraegen G, Claeys G, Meeus G, Delanghe M. Pseudomonas pickettii as a cause of pseudobacteremia. J Clin Microbiol 1985; 21:278-279.

6. Luk WK. An outbreak of pseudobacteraemia caused by Burkholderia pickettii: the critical role of an epidemiological link. J Hosp Infect 1996; 34: $59-69$. 